\title{
JXTA-Overlay P2P Platform and Its Application for Robot Control
}

\author{
Yuichi Ogata*, Evjola Spaho*, Keita Matsuo ${ }^{\dagger}$, Leonard Barolli ${ }^{\ddagger}$, Joan Arnedo-Moreno ${ }^{\S}$ and Fatos Xhafa \\ ${ }^{*}$ Graduate School of Engineering \\ Fukuoka Institute of Technology (FIT) \\ 3-30-1 Wajiro-Higashi, Higashi-Ku, Fukuoka 811-0295, Japan \\ Email:mgm10006@benefit.ac.jp,evjolaspaho@hotmail.com \\ ${ }^{\dagger}$ Fukuoka Prefectural Kaho-Sogo High School \\ 1117-1 Haji, Keisen-Machi, Kaho-Gun, Fukuoka 820-0607, Japan \\ E-mail: matuo-k7@fku.ed.jp \\ ${ }^{\ddagger}$ Department of Information and Communication Engineering \\ Fukuoka Institute of Technology (FIT) \\ 3-30-1 Wajiro-Higashi, Higashi-Ku, Fukuoka 811-0295, Japan \\ E-mail: barolli@fit.ac.jp \\ $\S$ Department of Computer Science, Multimedia and Telecommunication \\ Open University of Catalonia \\ Rambla del Poblenou, 15608018 Barcelona, Spain \\ E-mail: jarnedo@uoc.edu \\ "Department of Languages and Informatics Systems \\ Technical University of Catalonia \\ Jordi Girona 1-3, 08034 Barcelona, Spain \\ E-mail:fatos@lsi.upc.edu
}

\begin{abstract}
The design of an efficient collaborative multirobot framework that ensures the autonomy and the individual requirements of the involved robots is a very challenging task. This requires designing an efficient platform for inter-robot communication. P2P is a good approach to achieve this goal. P2P aims at making the communication ubiquitous thereby crossing the communication boundary and has many attractive features to use it as a platform for collaborative multi-robot environments. In this work, we present the JXTA Overlay P2P system and its application for robot control. Since JXTAOverlay is able to overcome Firewalls, Routers and NATs, it is possible to control end-devices in a WAN without changing the network security policy. We used JXTA-Overlay for the control of robot motors. We evaluated the proposed system by many experiments and have shown that the proposed system has a good performance and can be used successfully for robot control.
\end{abstract}

\section{INTRODUCTION}

Recently, many new devices and networks are appearing and in the past few years we have observed an explosive growth of multimedia computing, communication and applications.

The Internet is growing every day and the performance of computers is increased exponentially. However, the Internet architecture is based on Client/Server $(\mathrm{C} / \mathrm{S})$ topology, therefore can not use efficiently the clients features. It should be noted that computers of today have the same characteristics as old super-computers. Also, with appearance of new technologies such as ad-hoc networks, sensor networks, body networks, home networking, new network devices and applications will appear. Therefore, it is very important to monitor, control and optimize these network devices via communication channels. However, in large-scale networks such as Internet, it is very difficult to control the network devices, because of the security problems.

In order to make the networks secure many security devices are used. The firewalls are used for checking the information between private and public networks. The information is transmitted according to some decided rules and it is very difficult to change the network security policy. Also, there are many small networks and Intranets that do not allow the information coming from other networks. Therefore, recently many researchers are working on Peerto-Peer (P2P) networks, which are able to overcome the firewalls, NATs and other security devices without changing the network policy. Thus, P2P architectures will be very important for future distributed systems and applications. In such systems, the computational burden of the system can be distributed to peer nodes of the system. Therefore, in decentralized systems users become themselves actors by sharing, contributing and controlling the resources of 
the system. This characteristic makes $\mathrm{P} 2 \mathrm{P}$ systems very interesting for the development of decentralized applications [1], [2].

Robots are being steadily introduced into modern everyday life and are expected to play a key role in the near future. Typically, the robots are deployed in situations where it is too dangerous, expensive, tedious, and complex for humans to operate. Although many of the real-life applications may only need a single robot, a large number of them require the cooperation of a team of robots to accomplish a certain task. The use of multiple robots of overlapping capabilities offers a cost-effective and more robust solution. This redundancy in the robots' capabilities makes the overall system more flexible and fault-tolerant.

There are many research work for the control of single mobile robot, but not much has been done for cooperative mobile robots. The design of an efficient collaborative multi-robot framework that ensures the autonomy and the individual requirements of the involved robots is a very challenging task. This requires designing an efficient platform for inter-robot communication. $\mathrm{P} 2 \mathrm{P}$ is a good approach to achieve this goal. P2P aims at making the communication ubiquitous thereby crossing the communication boundary. It is network independent, scalable and can operate over TCP/IP, Bluetooth, and other wired and wireless technologies. It also facilitates creating any overlay network which provides an automatic and efficient abstraction of the physical network implementation details. As a result, $\mathrm{P} 2 \mathrm{P}$ technology shows many attractive features to use it as a platform for collaborative multi-robot environments [3].

In this paper, we present a P2P platform based on JXTA technology called JXTA-Overlay. We use JXTA-Overlay as a platform for robot control and communication.

The structure of this paper is as follows. In Section 2, we introduce the related work. In Section 3, we introduce the Project JXTA and JXTA-Overlay. In Section 4, we present the proposed $\mathrm{P} 2 \mathrm{P}$ robot control system. In section 5, we discuss the experimental result. Finally, conclusions and future work are given in Section 6.

\section{RELATED WORK}

In this section, we discuss the related work for P2P systems and their application for robot control and communication.

Much of the initial research efforts and projects on P2P systems were concerned with the investigation of overlay networks. It should be noted that each time more, this effort is oriented towards the design and implementation of libraries and platforms to support the development of real P2P applications, which could actually combine both Grid and P2P technologies. Bal et al. [4] motivated the need for research from conceptual, algorithmic and application level tools to facilitate the application development task, that is, tools that enable writing, deploying and running Grid/P2P applications.

Since in P2P applications file sharing plays an important role, a lot of research effort is being devoted to the devel- opment of reliable file sharing systems to be used in $\mathrm{P} 2 \mathrm{P}$ applications [5]. In particular, several studies are done for measuring the efficiency in $\mathrm{P} 2 \mathrm{P}$ systems [6], [7] as it is crucial.

Developing a multi-robot control architecture has been the subject of several studies. Asama et al. proposed ACTor-based Robot and Equipments Synthetic System (ACTRESS) [8]. The ACTRESS architecture tackles the issues of communication protocols with different abstraction levels, path planning, and task assignment through multi-stage communication protocols. The architecture was tested on heterogeneous mobile robots performing complex object pushing tasks that cannot be accomplished with a single robot.

In [9] is proposed a biologically inspired CEllular roBOTics system (CEBOT). Robots within the CEBOT architecture are tightly coupled and can dynamically reconfigure their physical structure in response to different environment changes. The CEBOT hierarchy is based on the selection of a cluster of master cells to coordinate subtasks by communicating with other master cells. Efficient communication protocols among CEBOT agents have also been discussed in several studies. Reducing the communication requirements, estimating the volume of information exchange between the involved robots, and effective solutions for choosing the master cells, were among the addressed issues [10].

GOFER is another distributed multi-robot problem solving architecture [11], [12]. It is based on a centralized task planning and scheduling module, which keeps track of the task allocation and the availability of all the robots through direct communication with them. The system also uses conventional artificial intelligence and task allocation algorithms, such as Contract Net Protocol to dispatch the subtasks to be performed by individual robots. Despite its satisfactory performance in applications, like following a wall and pushing objects, most of the GOFER implementations involved no more than three robots. In addition, GOFER is designed for indoor environments only and its dependence on a centralized task planning and scheduling module represents a major limitation from a flexibility and fault-tolerance perspective.

In order to alleviate the naturally inherited problems in most of the aforementioned framework, the ALLIANCE architecture was introduced [13]. This architecture emphasizes some key features in the design of multi-robot coordination architectures with a particular focus on faulttolerance, reliability, and adaptability. The architecture is designed for small to medium-sized teams of loosely coupled heterogeneous mobile robots operating in dynamic environments to perform tasks with possible ordering dependencies. The ALLIANCE architecture also assumes that the robots are probabilistically capable of assessing the performance of their actions as well as those of others through perception and broadcast communication.

There are some other research works that deal with the 


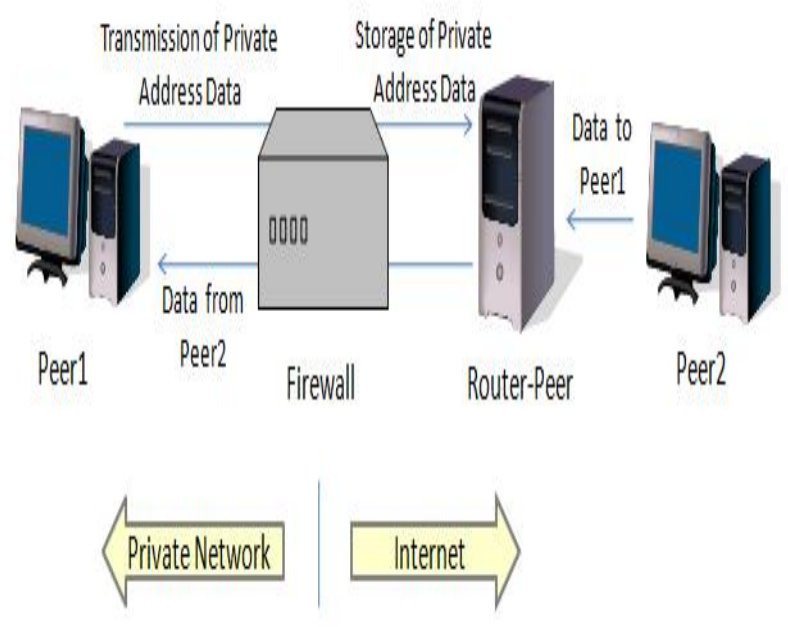

Figure 1. P2P communication.

autonomous distributed robot systems. In these systems, many autonomous mobile robots cooperate together to carry out complicated tasks [14], [15], [16], [17], [18]. By using many robots in a distributed way, the robot functions can be simplified and the system cost, fault tolerance and flexibility can be improved. In the case, where there are many robots and if one robot has a problem, the other robots can cooperate to finish a requested task. Therefore, the distributed robot systems are better than single robots. However, in distributed robot systems, to realise a common task, the robots should cooperate together and consider not only their own task but also the complete task.

In [19] is proposed an Adaptive Reservation-Time Division Multiple Access (AR-TDMA) medium access control protocol which can realise a real-time communication among robots in a heterogeneous environment by using a reservation mechanism. Also, by using an adaptive time slot allocation method, the protocol has a flexible behaviour and can deal with the changes of number of robots. Furthermore, the proposed protocol can reduce the packet collision probability.

However, all these research work deal with different communication protocols for robot communication. Our proposed platform includes a set of protocols that are able for robot communication and control. Our platform is based on JXTA architecture [20], [21], [22] and is implemented by Java Language. Thus, it is platform independent (does not depend on the OS).

\section{JXTA TECHNOLOGY AND JXTA-OVERLAY}

\section{A. JXTA Technology}

JXTA technology is a generalized group of protocols that allow different devices to communicate and collaborate among them. JXTA offers a platform covering basic needs in developing P2P networks.

By using the JXTA framework, it is possible that a peer in a private network can be connected to a peer in the Internet by overcoming existing firewalls as shown in Fig. 1. In this figure, the most important entity is the router peer. A router peer is any peer which supports the peer endpoint protocol and routing messages between peer in JXTA. The procedure to overcome the firewall is as follows.

- In the Router Peer is stored the private address of Peer1 by using the HTTP protocol to pass the firewall from Peer1.

- The Router Peer receives the data from Peer2 and access the Private address of Peer1 to transmit the data.

JXTA is an interesting alternative for developing P2P systems and groupware tools. In particular, it is appropriate for file sharing given that the protocols allow to develop either pure or mixed P2P networks. This last property is certainly important since pure $\mathrm{P} 2 \mathrm{P}$ systems need not the presence of a server for managing the network.

\section{B. JXTA-Overlay}

JXTA-Overlay project is an effort to use JXTA technology for building an overlay on top of JXTA offering a set of basic primitives (functionalities) that are most commonly needed in JXTA-based applications. The proposed overlay comprises the following primitives:

- peer discovery,

- peer's resources discovery,

- resource allocation,

- task submission and execution,

- file/data sharing, discovery and transmission,

- instant communication,

- peer group functionalities (groups, rooms etc.),

- monitoring of peers, groups and tasks.

This set of basic functionalities is intended to be as complete as possible to satisfy the needs of JXTA-based applications. The overlay is built on top of JXTA layer and provides a set of primitives that can be used by other applications, which on their hand, will be built on top of the overlay, with complete independence. The JXTAOverlay project has been developed using the ver-2.3 JXTA libraries. In fact, the project offers several improvements of the original JXTA protocols/services in order to increase the reliability of JXTA-based distributed applications [1] and to support group management and file sharing.

The architecture of the $\mathrm{P} 2 \mathrm{P}$ distributed platform we have developed using JXTA technology has two main peers: Broker and Client. Altogether these two peers form a new overlay on top of JXTA. The structure of JXTA-Overlay system is shown in Fig. 2.

\section{Internal Architecture of JXTA-Overlay}

Except Broker and Client peers, the JXTA-Overlay has also SimpleClient peers as shown in Fig. 3. The control layer interacts with the JXTA layer, and is divided into two parts: a lower part with functionality common to any kind of peer, and a higher part with functionality specific to Brokers and Clients. 


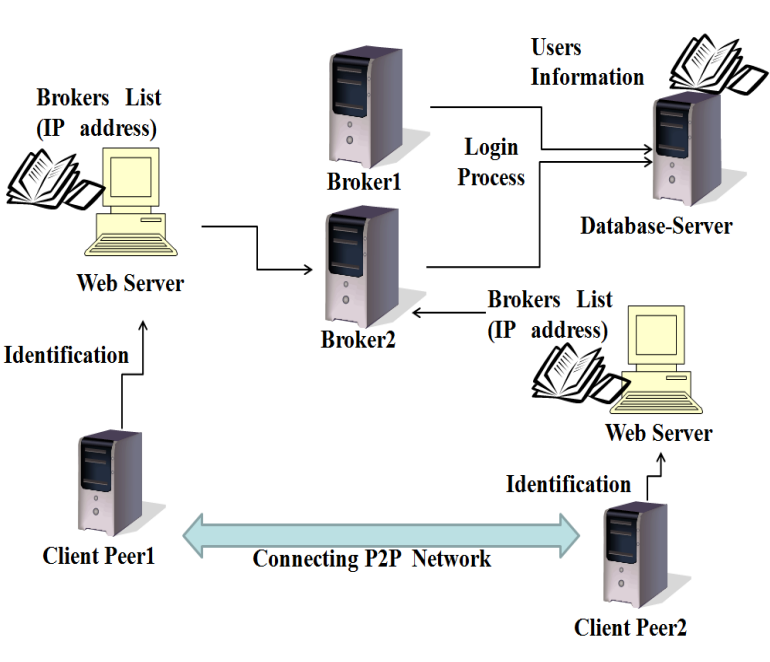

Figure 2. Structure of JXTA-Overlay system.

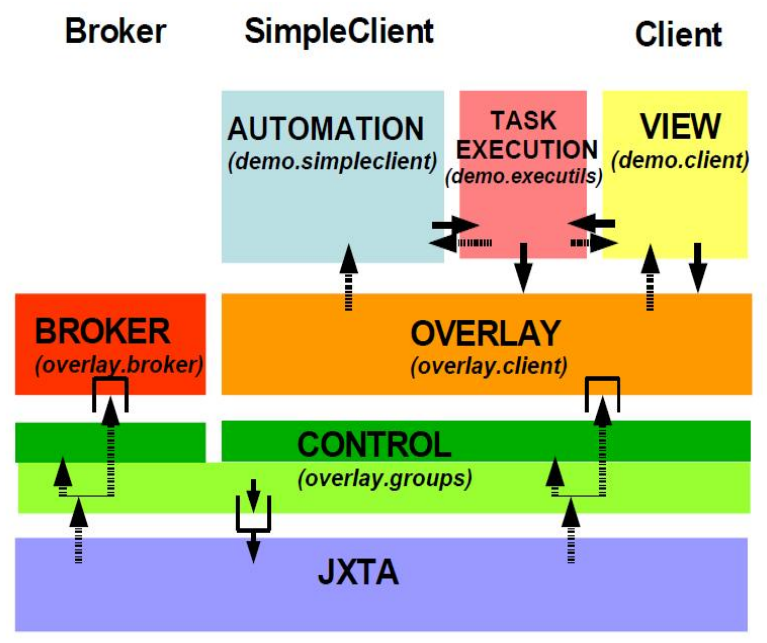

Figure 3. Internal Architecture of JXTA-Overlay.

- The common part provides functionality for doing JXTA messaging, discovery and advertisement.

- The Broker specific part provides functionality for managing groups of Brokers and keeping broker statistics.

- The Client specific part provides functionality for managing groups of Clients, keeping client statistics, managing its shareable files, managing the user configuration and creating the connection with a Broker.

The lower part enqueues the JXTA messages to be sent. Whenever a message arrives, the JXTA layer fires an event to the lower layer, which in turn fires a notifications to the upper layers.

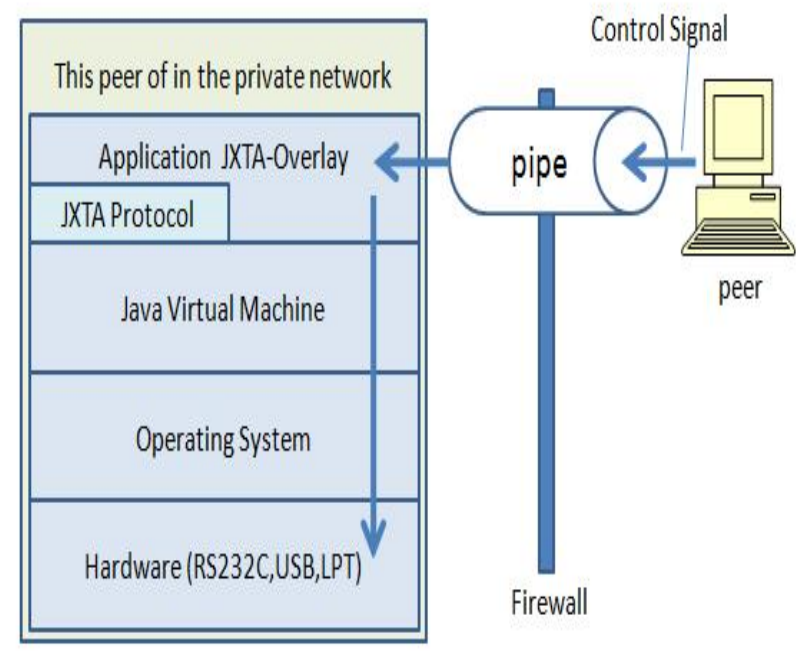

Figure 4. Implementation of remote control in JXTA-Overlay.

\section{Proposed P2P Robot Control System}

\section{A. Transmission Control and Management in JXTA-Overlay}

The most important part in $\mathrm{P} 2 \mathrm{P}$ systems is the communication between peers. By using the proposed JXTA-Overlay, it is possible to overcome, firewalls, routers, NATs, and bridges in the private networks. We explain in following the message transmission by the JXTA-Overlay.

JXTA-Overlay uses Universally Unique Identifier (UUID) in order to identify the peers in the private network from the Internet. The UUID is a general unique identifier. It is generated by the NIC address of the computer, date and time. By knowing the UUID and TCP address, it is possible to make address translation.

We implemented a control system that is able to control a peer in a private network from a peer in the Internet as showing in Fig. 4. The control targets are considered the network devices such as RS232C port, LPT port and USB port. By implementing this kind of control system, we are able to collect data and control the peers in a Wide Area Network (WAN). Thus, we will be able to control all devices that are connected to the peers. We control the USB devices and RS232C equipment. This is because USB devices are very popular and are used almost in every computer. Also, by using USB it is possible to control robot motors. The RS232C is a legal interface and many devices have implemented it.

\section{B. Implementation of P2P Robot Control System}

In our proposed system, we combine $\mathrm{P} 2 \mathrm{P}$ communication and robot technology. We implemented a robot control system as shown in Fig. 5. A snapshot of the robot control system is shown in 6. Our goal is not only to control the robots in a WAN but also to overcome the firewalls and other security devices. This is why we use the JXTAOverlay P2P platform. 


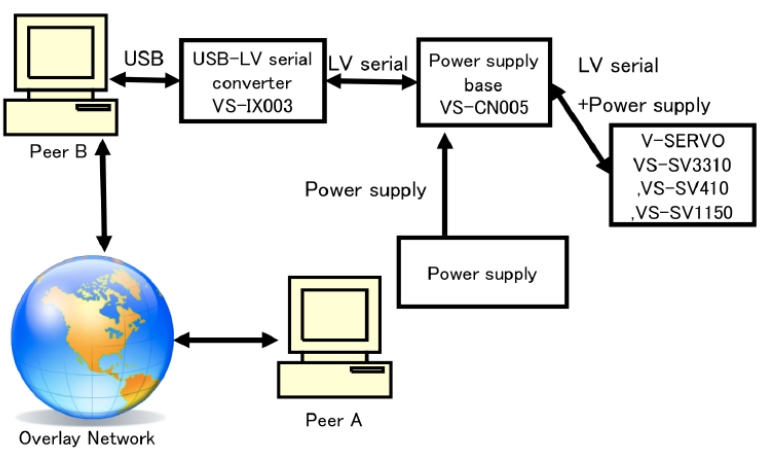

Figure 5. P2P Robot Control System.

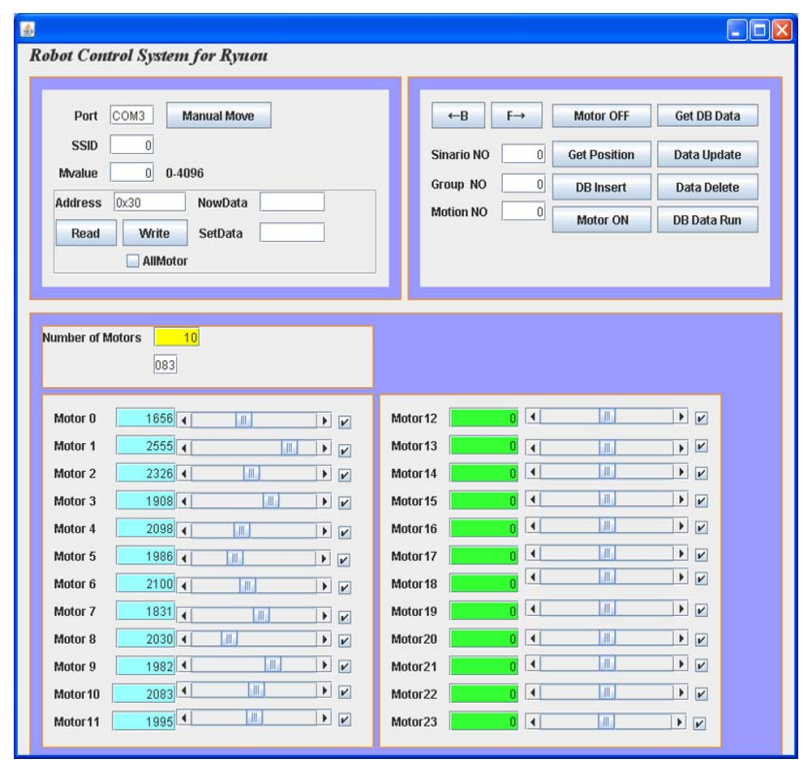

Figure 6. Screen of robot control system.

\section{EXPERIMENTAL RESUlts}

\section{A. Experiments with Robot Motors}

In these experiments, we controlled the Motor (VSERVO) of robot via JXTA-Overlay P2P system. We carried out experiments in real environment and confirmed the effectiveness of JXTA-Overlay. We measured the response time during robot control. The experimental data are shown in Fig. 7. Most of the response time data are less than one second, which is enough for the real control and communication.

\section{B. Experiments with Biped Walking Robot}

In Fig. 8 and Fig. 9, we show the control of KHR-1 robot by JXTA-Overlay for two cases: walking and greeting. The control interface for robot servo-motors is shown in Fig. 10. In Fig. 8, from the left to right, is shown the step movement of the robot and in Fig. 9 the greeting movement. From the right to the left, for greeting, the robot moves the hand, and then bows the body. We verified by experiments that the JXTA-Overlay controlled the robot in the smoothly way.

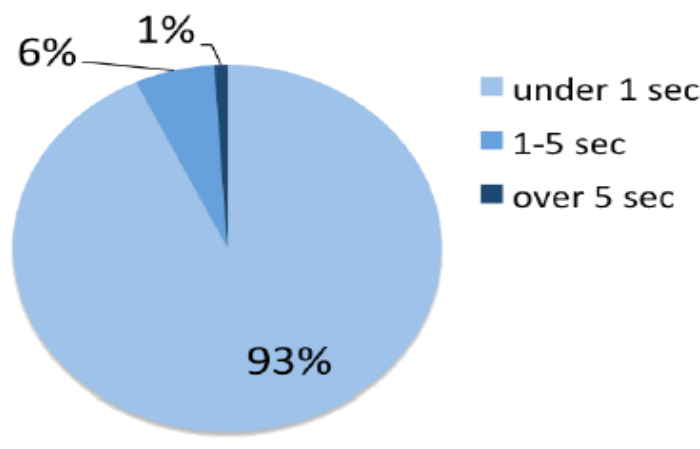

Figure 7. The response time of P2P JXTA-Overlay for robot control.

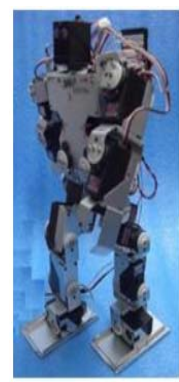

(a)

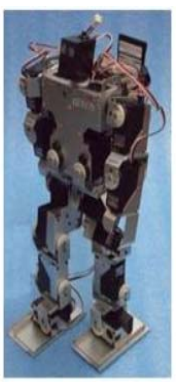

(b)

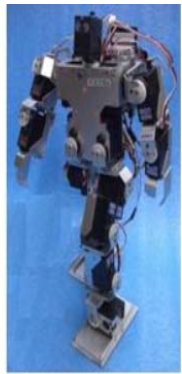

(c)



(d)
Figure 8. KHR-1 robot walking experiment.

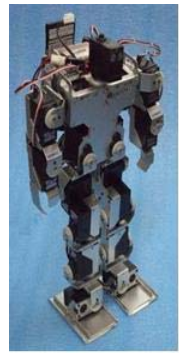

(a)

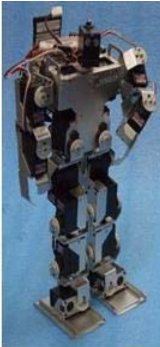

(b)

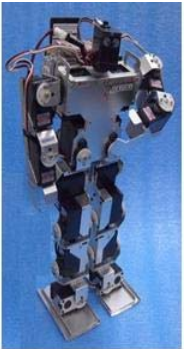

(c)

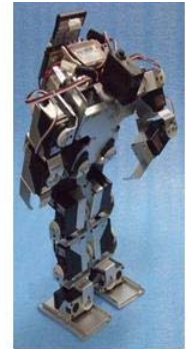

(d)
Figure 9. KHR-1 robot greeting movement.

\section{CONCLUSiOnS AND Future WORK}

In this work, we presented the JXTA Overlay P2P system and its application for robot control. The design, implementation and deployment of full featured P2P networks that integrate not only PCs and servers but also mobile and enddevices is very important for research in P2P systems. The findings of this work are a step forward in this direction.

Since JXTA-Overlay is able to overcome Firewalls, Routers and NATs, it is possible to control end-devices in a WAN without changing the network security policy. We used JXTA-Overlay for the control of robot motors. 


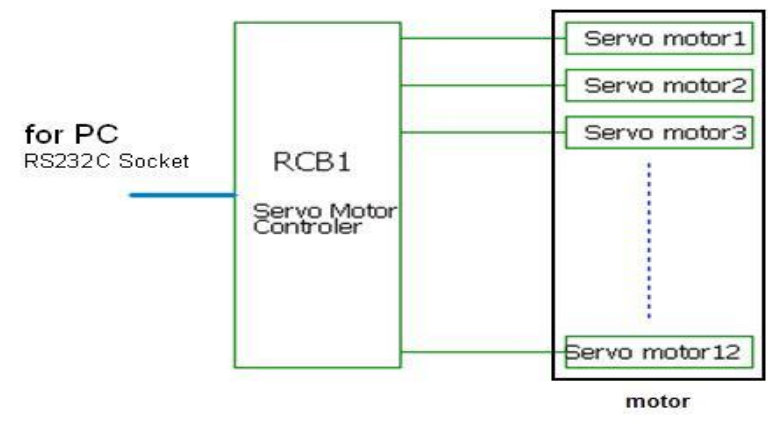

Figure 10. KHR-1 robot interface.

We evaluated the proposed system by many experiments and have shown that the proposed system has a good performance and can be used successfully for the control of robot.

In this work, we considered the robots as and-devices. In the future, we consider the robots as peers. We will implement JXTA-Overlay platform in real robots and carry out experiments in real environments.

\section{REFERENCES}

[1] F. Xhafa, R. Fernandez, T. Daradoumis, L. Barolli, S. Caballe, "Improvement of JXTA Protocols for Supporting Reliable Distributed Applications in P2P Systems", Proc. of NBiS-2007 (Regensburg, Germany), LNCS 4658, pp. 345354, September 2007.

[2] L. Barolli, F. Xhafa, A. Durresi, G. De Marco, "M3PS: A JXTA-based Multi-platform P2P System and Its Web Application Tools", International Journal of Web Information Systems, Vol. 2, No. 3/4, pp. 187-196, 2006.

[3] H. Asama, "Distributed Adaptive Robotics for Cosustainable Engineering", Journal of the Robotics Society of Japan, Vol. 20, No. 6, pp.577-578, 2002.

[4] H. Bal, H. Casanova, J. Dongarra, and S. Matsuoka, "Application-Level Tools", In Foster et. al. (Editors), The Grid: Blueprint for a New Computing Infrastructure, Chapter 24, pp. 463-489, Morgan Kaufmann, 2003.

[5] S. M. Lui and S.H. Kwok, "Interoperability of Peer-to-Peer File Sharing Protocols", SIGecom Exch. Journal, Vol. 3, No. 3, pp. 25-33, 2002.

[6] F. Le Fessant, S. Handurukande, A. M. Kermarrec, L. Massouli, "Clustering in Peer-to-Peer File Sharing Workloads", Peer-to-Peer Systems III, Lecture Notes in Computer Science, Vol. 3279, pp. 217-226, 2005.

[7] K. P. Gummadi, R. J. Dunn, S. Saroiu, S. D. Gribble, H. M. Levy, J. Zahorjan, "Measurement, Modeling, and Analysis of a Peer-to-Peer File-sharing Workload", Proc. of 19-th ACM Symposium on Operating Systems Principles, pp. 314-329, 2003.
[8] H. Asama, K. Ozaki, A. Matsumoto, Y. Ishida, and I. Endo, "Development of Task Assignment System Using Communication for Multiple Autonomous Robots", Journal of Robotics and Mechatronics, Vol.4, No.2, pp. 122-127, 1992.

[9] T. Fukuda and G. Iritani, "Construction Mechanism of Group Behaviour with Cooperation", Proc. of IEEE/RSJ IROSf95, pp. 535-542, 1995.

[10] T. Fukuda, Y. Kawauchi, and H. Asama, "Analysis and Evaluation of Cellular Robotics (CEBOT) as a Distributed Intelligent System by Communication Information Amount", Proc. of IEEE/RSJ IROS'90, pp. 827-834, 1990.

[11] P. Caloud, W. Choi, J. C. Latombe, C. Le Pape, and M. Yim, "Indoor Automation with Many Mobile Robots", Proc. of the IEEE International Workshop on Intelligent Robots and Systems, pp. 67-72, July 1990.

[12] C. Le Pape, "A Combination of Centralized and Distributed Methods for Multi-agent Planning and Scheduling", Proc. of the IEEE International Conference on Robotics and Automation, pp. 488-493, 1990.

[13] L. E. Parker, "Alliance: An Architecture for Fault Tolerant Multirobot Cooperation", IEEE Transactions on Robotics and Automation, Vol. 14, No. 2, pp. 220-240, April 1998.

[14] L. Chaimowicz, T. Sugar, V. Kumar, and M.F.M. Campos, "An Architecture for Tightly Coupled Multi-robot Cooperation", Proc. of IEEE International Conference on Robotics and Automation, pp. 2992-2997, 2001.

[15] L. Inoue, and T. Nakajima, "Cooperative Object Transportation by Multiple Robots with Their Own Objective Tasks", Journal of the Robotics Society of Japan, Vol. 19, No. 7, pp. 888-896, 2001.

[16] K. Ozaki, H. Asama, Y. Ishida, A. Matsumoto, I.Endo, "Collision Avoidance Using Communication Between Autonomous Mobile Robot", Journal of the Robotics Society of Japan, Vol. 14, No. 7, pp. 961-967, 1996.

[17] M. Parnichkun, S. Ozono, "CDCSMA-CD Communication Method for Cooperative Robot Systems", Advanced Robotics, Vol. 11, No. 7, pp. 669-694, 1998.

[18] P. E. Rybski, S. A. Stoeter, M. Gini, D. F. Hougen, and N. P. Papanikolopoulos, "Performance of a Distributed Robotic System Using Shared Communications Channels", IEEE Transactions on Robotics and Automation, Vol. 18, No. 5, pp. 713-727, 2002.

[19] J. Arai, A. Koyama, and L. Barolli, "AR-TDMA: An Adaptive Reservation Time Division Multiple Access Control Protocol for Robot Inter-communication", International Journal of Wireless and Mobile Computing (IJWMC), Vol. 3, No. 1/2, pp. 4-11, 2008.

[20] D. Brookshier, D. Govoni, N. Krishnan, J.C Soto, "JXTA: Java P2P Programming", Sams Publishing, 2002.

[21] IBM Corporation, "Making P2P Interoperable: Creating JXTA Systems Design P2P Systems that Extend Beyond Traditional Network Boundaries”, 2007.

[22] Sun Microsystems, "JXTA Java Standard Edition V2.5: Programmers Guide", June 2007. 